\title{
Inflammatory Cytokine response in a cohort of patients carrying novel NLRP12 variants
}

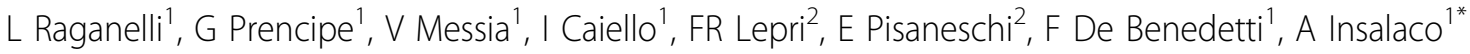 \\ From 8th International Congress of Familial Mediterranean Fever and Systemic Autoinflammatory Diseases \\ Dresden, Germany. 30 September - 3 October 2015
}

\section{Introduction}

The NLRP12 related autoinflammatory disorder (NLRP12$\mathrm{RD}$ ) is a rare autosomal dominant disease, caused by mutations in the NLRP12 gene, a member of NLRs family involved in negative regulation of NF-kB pro-inflammatory pathways. At present, few cases have been reported, although novel NLRP12 variants have recently been described.

\section{Objective}

To assess ex vivo the production of inflammatory cytokines, in patients carrying different NLRP12 variants not yet demonstrated as being associated to NLRP12-RD, in order to demonstrate the potential functional and pathogenic role of these variants.

\section{Materials and methods}

30 children, carrying NLRP12 variants in heterozygosis, and 1 patient, carrying the F402L variant in homozygosis, were identified using Next Generation Sequencing [G39V $(\mathrm{n}=16)$, F402L $(\mathrm{n}=8)$, H304Y $(\mathrm{n}=3)$, T260M $(\mathrm{n}=2)$ and G448A ( $n=1)$ ]; clinical information was collected for all patients. Patients with G39V were excluded from subsequent analysis, due to its high frequency both in our cohort $(17.4 \%)$ as in the general population. Whole blood cultures from NLRP12 patients and Juvenile Idiopathic Arthritis (JIA) patients ( $\mathrm{n}=10$, pathological control) were stimulated ex vivo with TLRs ligands (LPS 1-10-100 ng/mL, Zymosan $10 \mu \mathrm{g} / \mathrm{mL}$ ), with or without the further addition of ATP, for 5 or 22 hours. TNF- $\alpha$, IL- $1 \beta$ and IL- 6 were measured in supernatants by ELISA.

\section{Results}

Among all stimuli used, we observed a clear inflammatory response in cells stimulated with $10 \mathrm{mg} / \mathrm{ml}$ of

'Bambino Gesù Children Hospital, Pediatric Medicine-Rheumatology, Roma, Italy

Full list of author information is available at the end of the article
Zymosan. Indeed, we found that the TNF- $\alpha$ release, after stimulation with Zymosan, was higherboth at 5 and 22 hours of incubationin all NLRP12 patients, although carrying different variants, compared to JIA patients. Moreover, we observed a trend for higher IL-6 levels released from a significant number of NLRP12 patients both at 5 and 22 hours of incubation. Interestingly, we did not find differences in the IL- $1 \beta$ levels measured in media from NLRP12 patients, compared with JIA patients. When we investigated parents, carrying the same NLRP12 variant, we did not find any differences in cytokines released in response to stimulation with TLRs ligands, compared with those obtained from age-matched healthy donors. Moreover, no association of a specific clinical phenotype with different NLRP12 variants, and a particularcytokine release signature was observed.

\section{Conclusion}

We were not able to demonstrate a clear association of the investigated NLRP12 variants with the specific clinical phenotype or with the profile of pro-inflammatory cytokine released ex vivo. Other studies are needed before final conclusions on the pathogenic role of these NLRP12 variants can be drawn.

\section{Authors' details}

${ }^{1}$ Bambino Gesù Children Hospital, Pediatric Medicine-Rheumatology, Roma, Italy. ${ }^{2}$ Bambino Gesù Children Hospital, Cytogenetics and Molecular Genetics, Rome, Italy.

Published: 28 September 2015

doi:10.1186/1546-0096-13-S1-023

Cite this article as: Raganelli et al:: Inflammatory Cytokine response in a cohort of patients carrying novel NLRP12 variants. Pediatric Rheumatology 2015 13(Suppl 1):O23. 